\title{
Correction to: Benchmarking performance of machine and deep learning-based methodologies for Urdu text document classification
}

\author{
Muhammad Nabeel Asim ${ }^{1,2,4}$ (D) $\cdot$ Muhammad Usman Ghani ${ }^{3,4} \cdot$ Muhammad Ali Ibrahim $^{2,4}$. \\ Waqar Mahmood ${ }^{4} \cdot$ Andreas Dengel $^{1,2} \cdot$ Sheraz Ahmed $^{1}$
}

Published online: 27 October 2020

(C) Springer-Verlag London Ltd., part of Springer Nature 2020

\section{Correction to:}

\section{Neural Computing and Applications}

https://doi.org/10.1007/s00521-020-05321-8

The article [Benchmarking performance of machine and deep learning-based methodologies for Urdu text document classification], written by [Muhammad Nabeel Asim, Muhammad Usman Ghani, Muhammad Ali Ibrahim, Waqar Mahmood, Andreas Dengel and Sheraz Ahmed], was originally published electronically on the publisher's internet portal (currently SpringerLink) on [24 September 2020] with open access. With the author(s)' decision to step back from Open Choice, the copyright of the article changed on [10 October 2020] to (c) [Springer-Verlag London Ltd., part of Springer Nature] [2020] and the article is forthwith distributed under the terms of copyright.

Publisher's Note Springer Nature remains neutral with regard to jurisdictional claims in published maps and institutional affiliations.

The original article can be found online at https:// doi.org/10.1007/s00521-020-05321-8.

Muhammad Nabeel Asim

Muhammad_Nabeel.Asim@dfki.de

Muhammad Usman Ghani

usman.ghani@uet.edu.pk

Muhammad Ali Ibrahim ali.ibrahim@kics.edu.pk

Waqar Mahmood director@kics.edu.pk

Andreas Dengel

Andreas.Dengel@dfki.de

Sheraz Ahmed

Sheraz.Ahmed@dfki.de
1 German Research Center for Artificial Intelligence (DFKI) GmbH, 67663 Kaiserslautern, Germany

2 Technische Universität Kaiserslautern, 67663 Kaiserslautern, Germany

3 Department of Computer Science, University of Engineering and Technology (UET), Lahore, Pakistan

4 Intelligent Criminology Research Lab, National Center of Artificial Intelligence, Al-Khawarizmi Institute of Computer Science, UET, Lahore, Pakistan 